UK policy

\title{
Physicists get new research council
}

A new research council to fund users of large scientific facilities is being launched in the UK this month. The Science and Technology Facilities Council (STFC), which comes into effect on 1 April, has been formed from a merger of the Particle Physics and Astronomy Research Council (PPARC) and the Council for the Central Laboratory of the Research Councils (CCLRC). With an annual budget of about $£ 500 \mathrm{~m}$, the new organization will be responsible for all large facilities used by physical scientists in the UK. It will also handle the membership of international bodies such as CERN and the European Space Agency, and administer grants for particle physicists, astronomers and nuclear physicists.

The chief executive of the STFC, space scientist Keith Mason, says that the new body was created to give the UK more influence in international projects and to take advantage of "synergies" between the two former research councils. One area of obvious overlap, he points out, is accelerator technology. The STFC will now be responsible for access to particlephysics facilities such as the upcoming Large Hadron Collider at CERN, which used to be part of PPARC's remit, and multi-user accelerators previously run by the CCLRC, such as the ISIS neutron source and the Diamond synchrotron, both at the Rutherford Appleton Laboratory in Oxfordshire.

Mason, who was previously chief executive of PPARC, also hopes that the broad remit of the new body will allow industry to take advantage of a wide range of UK scientific research. He points to the formation of two "science innovation campuses" - at

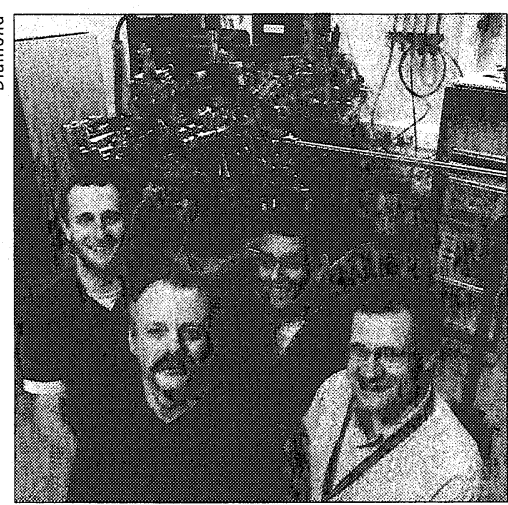

In mew hands Users of Diamond, like Chris Binns and colleagues from Leicester University, will now be funded by the Science and Technology Facilities Council.

Rutherford and the Daresbury laboratory in Cheshire - that are designed to improve the skills of workers within industry and attract investment from foreign companies.

While underlining the benefits of the new council, Mason acknowledges that he and his staff will need to be alert to the different needs of researchers who have used PPARC and CCLRC facilities. The former are often very familiar with the accelerators that they work on, while the latter only use accelerator facilities when they need them and also come from a wider range of scientific disciplines.

Susan Kilcoyne of Salford University, who chairs the ISIS user group, is concerned that researchers carrying out "small" science might lose out. For example, she wonders if the STFC could take money from ISIS users to pay for an unforeseen rise in the UK's CERN subscription. Mason admits there is "always a risk" that in exceptional circumstances money will have to be taken from one part of the council's budget to pay for a shortfall elsewhere, but, he says that he is "not afraid to take on that responsibility".

Another concern of Kilcoyne is that some users of central facilities such as ISIS will lose out because they are not given beam time by being awarded grants. The problem is that research councils are increasingly being required by government to cover "full economic costs" in their grant payments - i.e. to pay the cost of salaries and other overheads as well as specific project expenses. Universities may therefore be out of pocket if they employ central-facility users and consequently could decide not to take them on. Mason says that he is looking into ways to address this problem, perhaps by providing extra cash to users in proportion to the amount of beam time they are allocated.

Astronomers and particle physicists, meanwhile, seem happy with the new arrangements. It was originally mooted that researchers in these disciplines would be awarded grants by the Engineering and Physical Sciences Research Council (EPSRC) even though it would not have been looking after the running of their facilities. But this has been avoided after protests from the scientific community.

As for nuclear physicists, they are pleased to be joining the STFC. Until now they have been funded by EPSRC, which they believe often does not have the long-term perspective needed to fund large accelerator projects. However, Phil Walker, a nuclear physicist at Surrey University, is concerned that the handover is delaying a UK decision on whether to join the Facility for Antiproton and Ion Research (FAIR) that is being built in Germany. Mason says that the UK will join FAIR only if this is thought more scientifically important than a number of other large projects currently being considered. "We need to take our time to consider our options," he says. "The Germans are asking us to commit, but we have told them we need a bit of leeway on this." Edwin Cartidge

The new Science and Technology Facilities Council (STFC), meanwhile, inherits $\mathrm{E} 3 \mathrm{~m}$ worth of cuts from the Particle Physics and Astronomy Research Council. According to STFC chief executive Keith Mason, projects that have already been approved should go ahead but could be delayed. "Learning about this a month before the start of the financial year means that we cannot rearrange without causing pain," he says. "But we deal with it."

EPSRC interim chief executive Randal Richards hopes that the cuts are "a oneoff and that they will not happen again". Edwin Cartidge has more than doubled in real terms since the Labour party came to power in 1997.

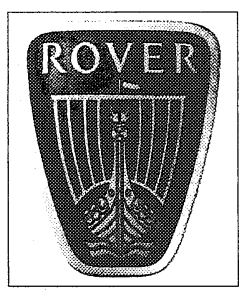

Knock-on effect Money to cover the Rover collapse has been taken from the UK science budget.
But scientists worry that this removal of supposedly "ring-fenced" funding sets a

The Engineering and Physical Scien esearch Council (EPSRC) will have taken from responsive-mode grants dided up pro rata among the different include cutting back on new beamlines at the recently completed Diamond synchrotron source and the cancellation of new nanotechnology equipment. 
CM-PRS00001634

2686821 